\title{
LDL cholesterol response to statins and future risk of cardiovascular disease
}

Ralph Kwame Akyea, Joe Kai, Nadeem Qureshi, Barbara Iyen, Stephen F Weng

\section{Correspondence}

To the Editor

Our recently published study using electronic health records from primary care setting in the UK examined low-density lipoprotein cholesterol (LDL-C) reduction among patients initiating statins. 1 We are grateful to Dr Taher Modarressi, Dr Dieter Lütjohann, Professor Oliver Weingärtner, Dr Tülin Muggleton and Dr Ellis Muggleton for their comments.

With regards to the points raised:

Suboptimal cholesterol response to initiation of statins and future risk of cardiovascular disease - Dr Taher Modarressi

While biomarkers are useful to establish associations with various disease conditions including cardiovascular disease (CVD), often these biomarkers are not routinely offered or measured in primary care settings within the UK, and hence are not available in electronic health records. Lipoprotein(a) $(\operatorname{Lp}(a))$, a large lipoprotein particle produced in the liver, is not routinely measured in patients in UK general practice. There is, however, evidence indicating $L p(a)$ might be a useful biomarker that has a causal link to atherosclerosis and associated with cholesterol-lowering response and CVD risk.2 More research is required to evaluate the adoption and clinical utility of $1 \mathrm{Lp}(\mathrm{a})$ in routine primary care settings.

High cholesterol absorption and response to statin therapy - Dr Dieter Lütjohann and Professor Oliver Weingärtner

As rightly indicated and observed in ' $4 S^{\prime}$ ' 3 and 'HIJ-PROPER' 4 trials, there is a need for individualised cholesterol-lowering therapies, and cholesterol absorption has a particular role in indicating specific types of cholesterol-lowering therapies. In addition to biological markers for cholesterol absorption, common genetic variants identified in by the SEARCH consortium, 5 such as SLC01B1, also indicate that genetics may have a role to play in simvastatin-induced myopathy. These complex factors rightly indicate the need for personalised therapies. Critically, improving monitoring of LDL-C response and consideration of ongoing titration or alternative medications for patients initiated on statin therapy will help ensure appropriate management can be implemented to achieve the recommended reductions in LDL-C.6 7

LDL-C consists of different subclasses, more precise diagnostics are required - Dr Tülin Muggleton and Dr Ellis Muggleton

We acknowledge the importance of all-cause mortality; however, the outcomes of our study were only focused on major adverse cardiac outcomes (MACE), including CVD mortality. There is strong evidence to show that reduction in lipids will reduce both nonfatal and fatal CVD from randomised trials. 8 However, evidence on the effects of statins on non-CVD mortality is uncertain because this is extremely complex. 9 The current study was not designed and powered to evaluate this association between statin response and non-CVD mortality.

Although low-density lipoprotien ( $L D L$ ) in the form of small dense LDL (sdLDL) is associated with elevated levels of apolipoprotein B and is more likely to lead to the This article has been accepted for publication in Heart 2019 following peer review, and the Version of Record can be accessed online at http://dx.doi.org/10.1136/heartjnl-2019-315461

(c) Authors (or their employer(s) 2019

https://creativecommons.org/licenses/by-nc/4.0/ 
development of atherosclerosis, $1011 \mathrm{sdLDL}$ is also not routinely measured in UK primary care. Per cent change in LDL-C, therefore, remains the most widely measured surrogate measure of statin response.

Furthermore, primary care electronic health records have no data on medication adherence. Non-adherence was offered as a possible explanation for suboptimal response in our study. Poor adherence to statins and other medications administered for chronic conditions have been reported in a number of studies.12 As a research team, we are actively investigating the side effect profile and other factors such as polypharmacy, comorbidities and medication interactions that might potentially be related to suboptimal response.

Finally, we agree that considering the reference group as the suboptimal responders mean that optimal responders have reduced risk of cardiovascular disease. In fact, this interpretation is supported in the supplemental analysis in our original paper (online supplementary appendix 5)1 that a reduction of $1 \mathrm{mmol} / \mathrm{L}$ results in a $6 \%$ reduction in the risk of MACE outcomes. However, it remains factual and statistically correct as well that those who do not respond are at increased risk of CVD when compared with those who do not achieve recommended reductions in LDL-C.

\section{References}

1. Akyea RK, Kai J, Qureshi N, et al. Sub-optimal cholesterol response to initiation of statins and future risk of cardiovascular disease. Heart 2019;105:975-81

2. Willeit P, Ridker PM, Nestel PJ, et al. Baseline and on-statin treatment lipoprotein(a) levels for prediction of cardiovascular events: individual patient-data meta-analysis of statin outcome trials. Lancet 2018;392:1311-20 doi:10.1016/S01406736(18)31652-0

3. Miettinen TA, Gylling $H$, Strandberg $T$, et al. Baseline serum cholestanol as predictor of recurrent coronary events in subgroup of Scandinavian simvastatin survival study. Finnish 4S Investigators. BMJ 1998;316:1127-30 doi:10.1136/bmj.316.7138.1127

4. Yamaguchi J, Kawada-Watanabe E, Koyanagi R, et al. Baseline serum sitosterol level as predictor of adverse clinical events in acute coronary syndrome patients with dyslipidaemia: A sub-analysis of HIJ-PROPER. Atherosclerosis 2018;274:139-45 doi: $10.1016 / j$.atherosclerosis.2018.04.036

5. Link E, Parish S, Armitage J, et al. SLCO1B1 variants and statin-induced myopathy--a genomewide study. New England Journal of Medicine 2008;359:789-99 doi:10.1056/NEJMoa0801936

6. Stone NJ, Robinson JG, Lichtenstein AH, et al. ACC/AHA Guideline on the treatment of blood cholesterol to reduce atherosclerotic cardiovascular risk in adults a report of the American College of Cardiology/American Heart Association task force on practice guidelines. Circulation 2013;2014:S1-45

National Institute for Health and Care Excellence. Cardiovascular disease: risk assessment and reduction, including lipid modification. London: National Institute for Health and Care Excellence, 2016.

7. Law MR, Wald NJ, Rudnicka AR. Quantifying effect of statins on low density lipoprotein cholesterol, ischaemic heart disease, and stroke: systematic review and meta-analysis. BMJ 2003;326:1423 doi:10.1136/bmj.326.7404.1423

8. Singh M, Stewart R, White $H$. Importance of frailty in patients with cardiovascular disease. European Heart Journal 2014;35:1726-31 doi:10.1093/eurheartj/ehu197

9. Crouse JR, Parks JS, Schey HM, et al. Studies of low density lipoprotein molecular weight in human beings with coronary artery disease. Journal of Lipid Research $1985 ; 26: 566-74$ 
10. Ravnskov U, de Lorgeril M, Diamond DM, et al. LDL-C does not cause cardiovascular disease: a comprehensive review of the current literature. Expert Review of Clinical Pharmacology 2018;11:959-70 doi:10.1080/17512433.2018.1519391

11. Colantonio LD, Rosenson RS, Deng $L$, et al. Adherence to statin therapy among US adults between 2007 and 2014. Journal of the American Heart Association 2019;8: e010376 doi: $10.1161 /$ JAHA.118.010376

\section{Footnotes:}

RKA and SFW contributed equally.

Contributors: SFW is guarantor.

Funding: The research was funded and supported by the University of Nottingham.

Competing interests: NQ is a member of the National Institute for Health and Care Excellence (NICE) Familial Hypercholesterolaemia Guideline Development Group (CG71) and NICE Lipid Modification Guidelines Group (CG181). SFW is a member of the Clinical Practice Research Datalink Independent Scientific Advisory Committee and previously held an National Institute for Health Research - School for Primary Care Research (NIHRSPCR) career launching fellowship award. The remaining authors have no competing interests.

Provenance and peer review: Not commissioned; internally peer reviewed. 